\title{
Biomechanical evaluation of a customized 3D-printed polyetheretherketone condylar prosthesis
}

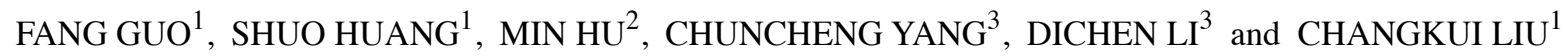 \\ ${ }^{1}$ Department of Oral and Maxillofacial Surgery, School of Stomatology, Xi'an Medical University, \\ Xi'an, Shaanxi 710021; ${ }^{2}$ Department of Oral and Maxillofacial Surgery, General Hospital of PLA, Beijing 100853; \\ ${ }^{3}$ State Key Laboratory for Manufacturing Systems Engineering, Xi'an Jiaotong University, Xi'an, Shaanxi 710054, P.R. China
}

Received March 16, 2020; Accepted January 22, 2021

DOI: $10.3892 /$ etm.2021.9779

\begin{abstract}
The present study aimed to evaluate the biomechanical behavior of a custom 3D-printed polyetheretherketone (PEEK) condylar prosthesis using finite element analysis and mechanical testing. The Mimics software was used to create a 3D model of the mandible, which was then imported into Geomagic Studio software to perform osteotomy of the lesion area. A customized PEEK condyle prosthesis was then designed and the finite element model of the PEEK condyle prosthesis, mandible and fixation screw was established. The maximum stress of the prosthesis and screws, as well as stress and strain of the cortical and cancellous bones in the intercuspal position, incisal clench, left unilateral molar clench and right unilateral molar clench was analyzed. The biomechanical properties of the prosthesis were studied using two models with different lesion ranges. To simulate the actual clinical situation, a special fixture was designed. The compression performance was tested at $1 \mathrm{~mm} / \mathrm{min}$ for the condyle prosthesis, prepared by fused deposition modeling (FDM). The results of a finite element analysis suggested that the maximum stress of the condyle was $10.733 \mathrm{MPa}$ and the maximum stress of the screw was $9.7075 \mathrm{MPa}$; both were far less than the yield strength of the material. The maximum force that the two designed prostheses were able to with-

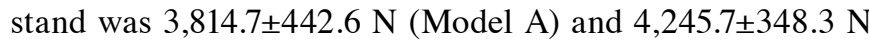
(Model B). Overall, the customized PEEK condyle prostheses prepared by FDM exhibited a uniform stress distribution and good mechanical properties, providing a theoretical basis for PEEK as a reconstruction material for repairing the temporomandibular joint.
\end{abstract}

Correspondence to: Professor Changkui Liu, Department of Oral and Maxillofacial Surgery, School of Stomatology, Xi'an Medical University, 1 Xinwang Road, Xi'an, Shaanxi 710021, P.R. China E-mail: dentistlck@126.com

Key words: custom design, polyetheretherketone, condylar prosthesis, finite element analysis, biomechanical analysis, 3D printing

\section{Introduction}

The temporomandibular joint (TMJ) is a bilaterally linked joint in the jaw that functions as a single entity when speaking, chewing, swallowing and yawning. More than $25 \%$ of the world's population is affected by temporomandibular joint disorders (TMDs) (1), leading to mandibular dysfunction and pain. TMJ replacement is recommended for $2-5 \%$ of TMDs linked to ankylosis, tumors and degenerative diseases (1). The goal of TMJ replacement is to reconstruct the anatomy and function of the mandible, enable the patient to resume a sustainable diet, prevent further morbidity, reduce pain and improve quality of life. In arthroplasty, transplantation with either autologous or allogeneic bone has substantial disadvantages when compared to artificial joint transplantation. These include limited supplies, limited dimensions and the risk of infection (bacterial or viral) $(2,3)$. Therefore, using an artificial TMJ for joint reconstruction is one of the most effective methods for clinical replacement.

Joint substitute implant materials require unique properties, including excellent biological properties, such as compatibility and safety, strong mechanical properties and fatigue resistance, a Young's modulus similar to that of human bone, biological inertia and good bio-surface modification capability. Orthopedic implant materials are typically composed of metals, inorganic non-metals (or bioceramics), polymers, complexes and biological derivatives. The most common orthopedic or dental materials are metals, such as titanium and precious metals that have excellent environmental resistance and good physical and biological properties (4). However, metallic materials may lead to various problems after implantation, including the release of toxic metal ions, interference with imaging diagnoses, bone resorption, osteolysis, allergic reactions and implant shedding $(5,6)$. In dentistry, the clinical use of precious metals, such as titanium, has resolved certain issues related to the use of metallic materials; however, the inherently high elastic modulus of metallic materials frequently leads to stress shielding due to mismatch with the human bone, resulting in bone absorption (7). At present, there are two types of mature TMJ prostheses worldwide, provided by the companies TMJ Concepts and Biomet Microfixation, whose condylar prostheses are predominantly made of metal. Therefore, there is still a requirement to improve the clinical 
performance of the prosthesis and reduce the incidence of post-operative complications by using more suitable biomaterials and subject-specific designs.

Polyetheretherketone (PEEK) is widely used in medical fields, such as cranioplasty (8-10) and spinal fusion $(11,12)$ for its light weight, excellent biocompatibility, biomechanical properties and stability. Specifically, its modulus of elasticity is similar to that of cortical bone, which improves load sharing between the implant and the surrounding tissue (13). However, reports on the use of PEEK in TMJ reconstruction are limited (14), primarily those evaluating the effect of carbon fiber reinforced-PEEK material in the reconstruction of TMJ defects in rabbits, and there is no relevant research on the biomechanical properties of PEEK materials, to the best of our knowledge. The objective of the present study was to evaluate the biomechanical behavior of custom 3D-printed PEEK condylar prostheses. For this purpose, a finite element analysis (FEA) was established to explore the effect of the clenching tasks on the prosthesis and surrounding bones. The designed prostheses were prepared using fusion deposition modeling (FDM) technology and their compression properties were tested using uniaxial compression experiments.

\section{Materials and methods}

Modeling the mandible and custom condylar prosthesis. In the present study, the skull of the patient was scanned CT (slice thickness, $0.625 \mathrm{~mm}$; slice interval, $0.5 \mathrm{~mm}$ ) for 26 dental implants at the Radiology Department of the First Affiliated Hospital of Xi'an Jiaotong University (Xi'an, China). The patient presented with a normal occlusion and without TMJ disease. The patient provided informed consent. The data were stored and then imported into the Mimics medical imaging processing software (version 20.0; Materialise, Inc.) for 3D reconstruction. In this modeling process, the mandible included the cortical bone, cancellous bone and complete dentition.

Due to the different extents of surgical osteotomy in real clinical applications, two models were created. One model (Model A) had a higher osteotomy position and retained the coracoid process, whereas the other model (Model B) had a lower osteotomy position and did not retain the coracoid process (Fig. 1). To design the corresponding prostheses and create the two models, the 3D reconstruction model of the mandible was imported into Geomagic Studio (version 2012; 3D Systems, Inc.). The osteotomy of the mandibular lesion was simulated according to the surgical requirements and partial bone grinding (circular blunt) was performed on the buccal side of the surgical stump to reduce the stress concentration on the corresponding part of the prostheses. The corresponding data of the contralateral mandible were symmetrically mapped to the defect area according to the natural symmetry of the mandibular side. Part of the condylar prostheses data in the defect area was obtained and the overall design of the prostheses was achieved (Fig. 1). The TMJ condylar prostheses were designed on the left side of the mandible and fixed with five screws (Fig. 1). Each screw was $8 \mathrm{~cm}$ in length, $2 \mathrm{~mm}$ in diameter and did not have any screw teeth.

FEA of the PEEK condylar prostheses. The FEM was used to investigate the biomechanical properties of the condylar pros- theses and mandible. In the present study, FEA was performed using the ANSYS Workbench finite element package (version 14.5; ANSYS, Inc.). The parameters of the materials used in the FEA are presented in Table I $(15,16)$. To reduce the influence of manufacturing methods and process parameters, the material properties of the PEEK condylar prostheses were defined based on the results of tensile, compression and flexure tests, which were conducted using an electrohydraulic servo mechanical testing machine (SANS CMT4304; MTS Corp.) according to ISO 527-1:2012, ISO 604 and ISO 178, respectively. All materials were assumed to be homogeneous, linearly elastic and isotropic.

Mesh Model A contained 1,157,007 nodes (778,460 elements) and mesh Model B contained 1,115,658 nodes (751,727 elements). The contact interface between the prosthesis and mandible was considered bonded. The loading conditions pertained to six principal muscles (Fig. 2). A total of four static clenching tasks were simulated in this study: The intercuspal position (ICP), incisal clench (INC), left unilateral molar clench (LMOL) and right unilateral molar clench (RMOL). The models were constrained in all directions at the top condyle in all clenching tasks. In addition, the models were constrained in the incisor regions (INC constraint), the canine and premolar regions (ICP constraint), the right molars (RMOL constraint) and the left molars (LMOL constraint). The magnitude and directions of the six muscular forces were obtained from a previous study (17). The maximum stress of the prostheses and screws and the stress and strain of the cortical bone and cancellous bones during ICP, INC, LMOL and RMOL were recorded and analyzed.

Preparation and mechanical testing of the condylar prostheses. The condylar prostheses were prepared using a self-developed 3D printer (Fig. 3A) with a nozzle diameter of $0.4 \mathrm{~mm}$, a printing speed of $40 \mathrm{~mm} / \mathrm{sec}$, a wall thickness of $0.8 \mathrm{~mm}$ (printing line width of $0.4 \mathrm{~mm}$ ), a layer thickness of $0.2 \mathrm{~mm}$, a nozzle temperature of $420^{\circ} \mathrm{C}$, an infill percentage of $100 \%$ and printing trajectories of tiled scan, buccal up and lingual down (Fig. 3B).

To evaluate the compression performance of the printed samples, a compression test was conducted for the 3D-printed condylar prostheses using a universal testing machine. The fixture was designed and fixed with titanium nails to simulate an actual clinical prosthesis (Fig. 3C). The fixed end of the fixture was clamped and the speed was tested at $1 \mathrm{~mm} / \mathrm{min}$ (Fig. 3F). A total of five physical models were used for the compression tests (Fig. 3D). Finally, the results of the FEA and experimental tests were compared to comprehensively assess the mechanical properties of the condylar prostheses.

\section{Results}

General. In the present study, the following parameters were evaluated: The von Mises stress of the condylar prosthesis and five bone screws along with the von Mises stress and von Mises strain of the cortical bone and cancellous bone in four grasping tasks. Figs. 4 and 5 provide the simulation results of the INC task for model A. Tables II and III present the evaluation parameters for all four occlusion tasks for the two models. 
Table I. Mechanical parameters of the materials used in the modeling.

\begin{tabular}{lcccc}
\hline Parameter & Cortical bone & Cancellous bone & Titanium alloy & PEEK \\
\hline Elastic modulus/GPa & 13 & 1.6 & 110 & 2.8 \\
Poisson's ratio & 0.3 & 0.3 & 0.3 & 0.3 \\
\hline
\end{tabular}

PEEK, polyetheretherketone.
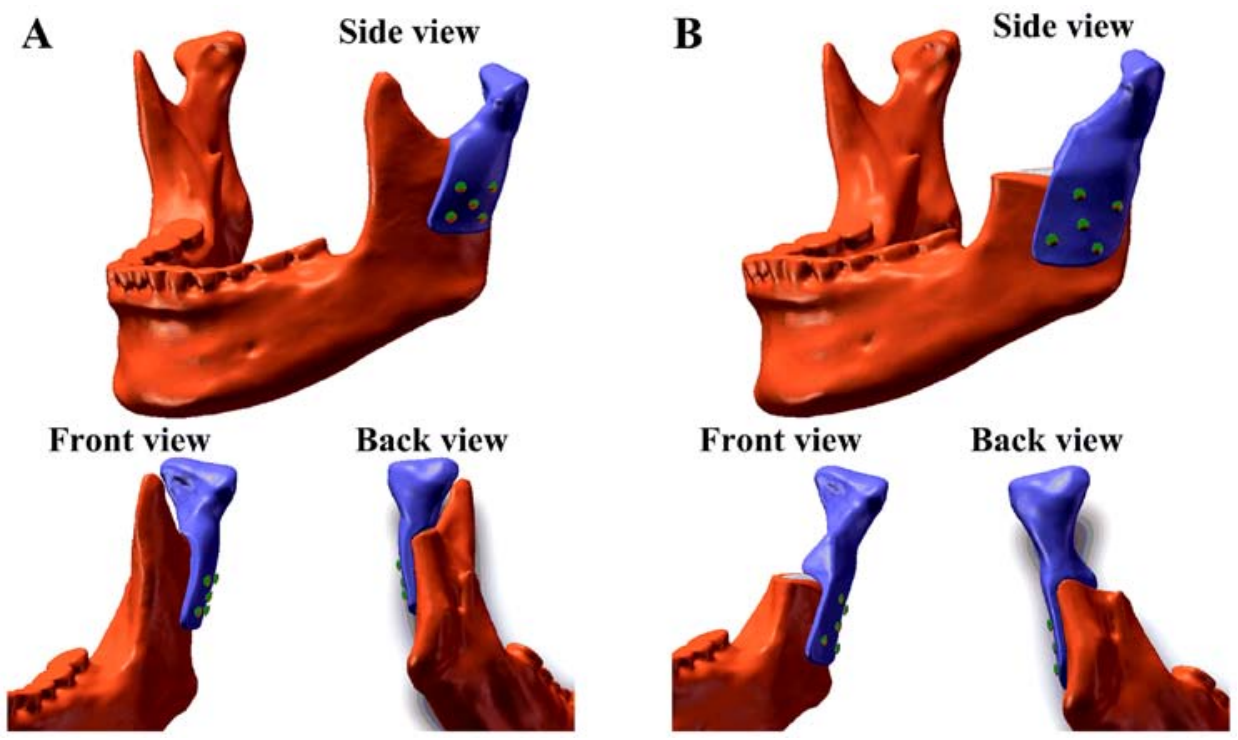

Figure 1. Assembly models of the polyetheretherketone condyle prostheses. (A) Model A and (B) Model B.

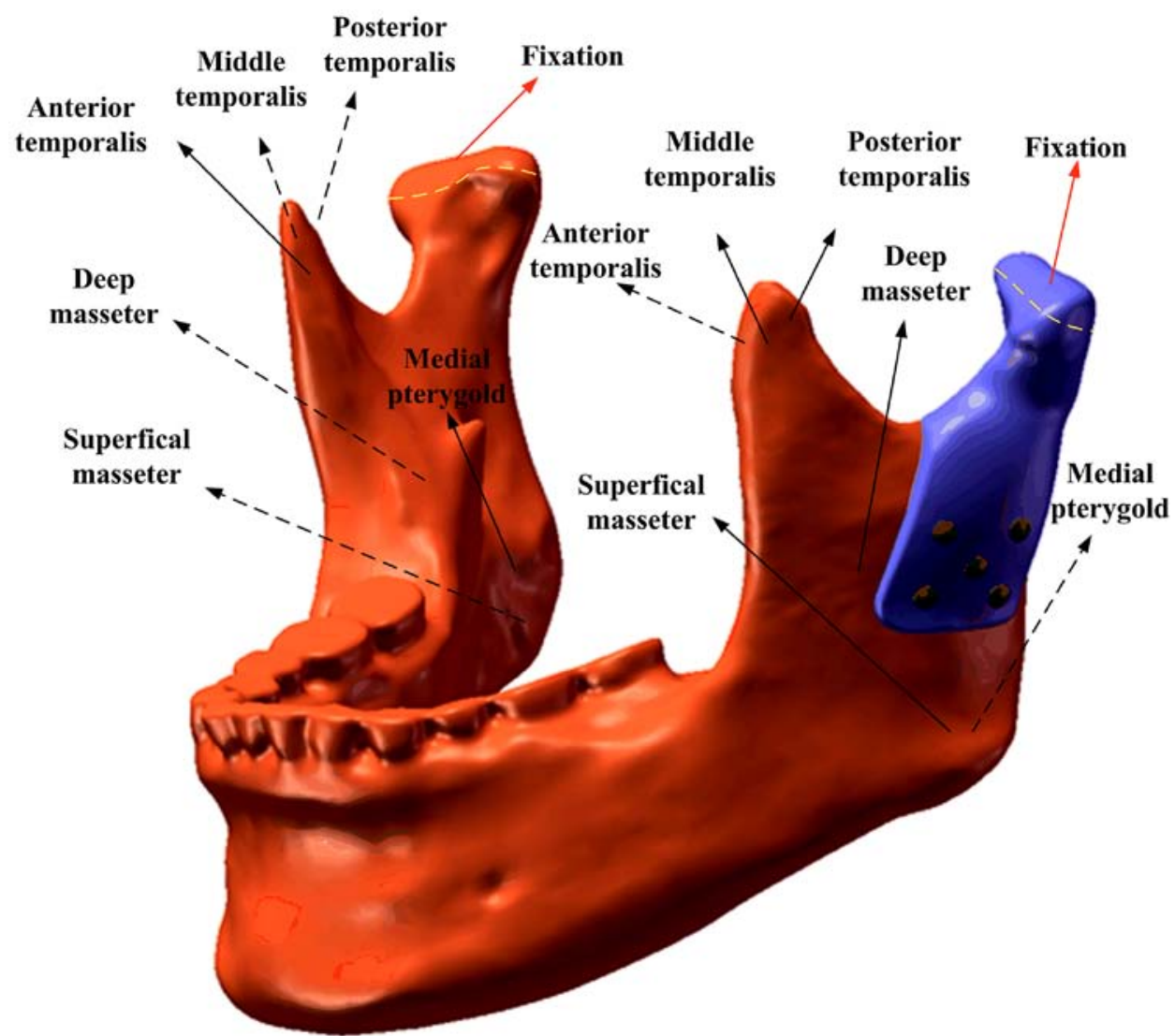

Figure 2. Muscular forces and constraints applied in finite element simulation. 

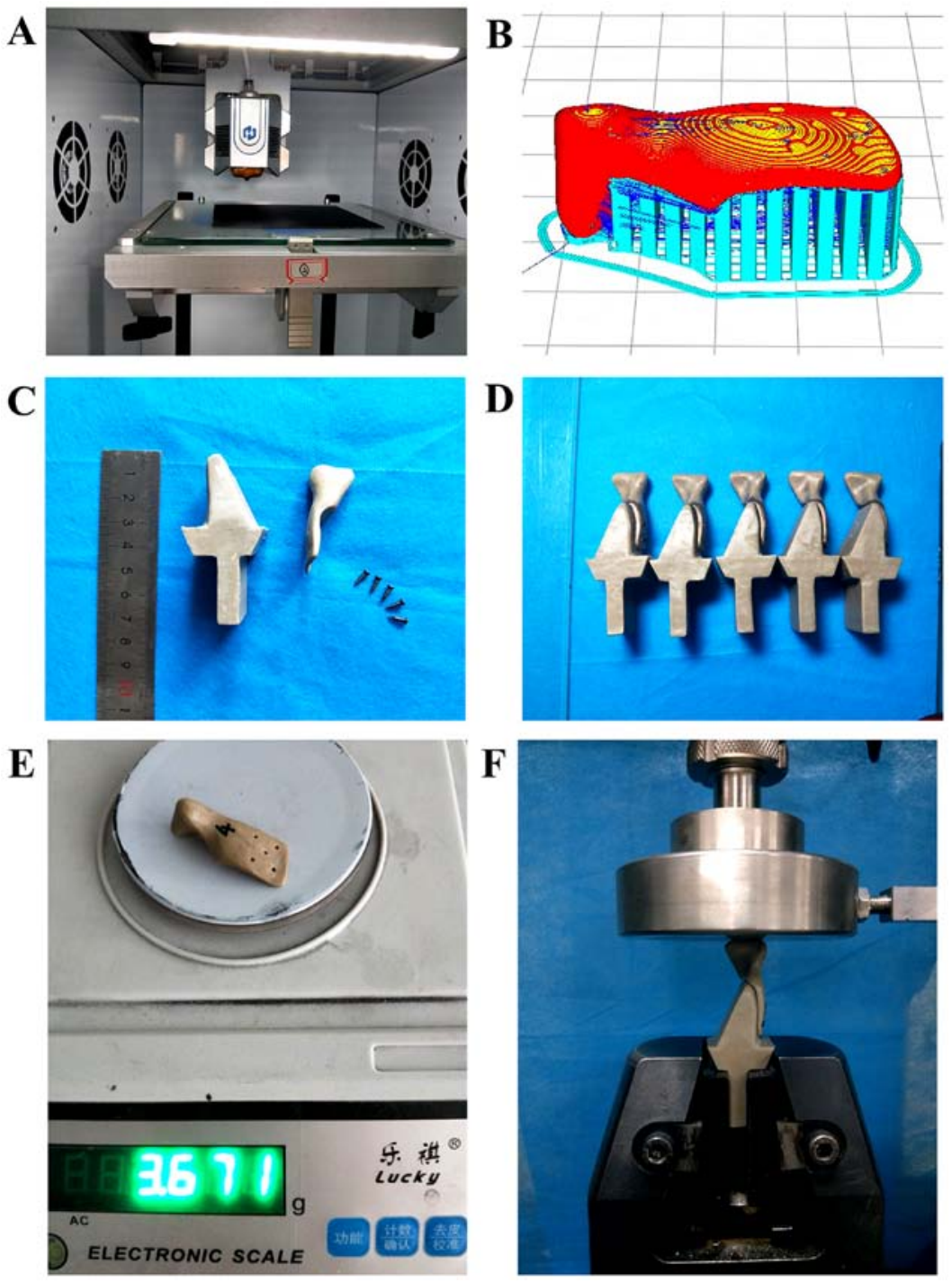

Figure 3. Preparation and mechanical testing of PEEK condylar prosthesis. (A) The 3D-printing device; (B) the printing direction of the prosthesis and the design of the print support (red, prosthesis; blue, support); (C) the custom condylar prosthesis and fixture; (D) assembled customized condyles and fixtures (side view); (E) weight of the PEEK condylar prosthesis (A model); and (F) configuration of the compression test. PEEK, polyetheretherketone.

Evaluation of von Mises stress of the TMJ condylar prosthesis and five bone screws. Mechanical analysis indicated that the stress values of prosthesis Model A were $<11 \mathrm{MPa}$ in all four clenching tasks. The von Mises stress on the five bone screws ranged from 3.07 MPa (INC, screw \#1) to $9.71 \mathrm{MPa}$ (ICP, screw \#5) for the same tasks. Scre \#5 was under the greatest von Mises stress during all tasks, as shown in Table II. For Model B, stress values of prosthesis were $<9 \mathrm{MPa}$ in all four occlusion tasks. The von Mises stress on the five bone screws ranged from $0.27 \mathrm{MPa}$ (LMOL, screw \#4) to $2.77 \mathrm{MPa}$ (RMOL, screw \#2) and screw \#2 was under the greatest stress during all tasks, as shown in Table III.

Evaluation of the von Mises stress and strain of the cortical and cancellous bones. The mechanical analysis of the entire mandible cortical bone in Model A suggested that the maximum and minimum von Mises stress occurred during the ICP (42.688 MPa) and INC (20.669 MPa) tests. The maximum and minimum von Mises strain also occurred during the ICP $(4,897.2 \mu)$ and INC $(1,781.5 \mu)$ tests. However, in the cancellous bone, the maximum and minimum von Mises stress and strain occurred during the ICP (10.826 MPa and 7,772.4 $\mu$, respectively) and LMOL (3.7015 MPa and 2,920.5 $\mu$, respectively) tests, as shown in Table II. Conversely, for the entire mandible cortical bone in Model B, the maximum and minimum von Mises stress occurred during the LMOL (43.038 MPa) and INC (13.93 MPa) tests, as did the maximum and minimum von Mises strain (4,066.1 and 1,240.4 $\mu$, respectively). In the cancellous bone, the maximum and minimum von Mises stress and strain occurred during the RMOL (3.4237 MPa and $2,277.5 \mu$, respectively) and LMOL $(2.4105 \mathrm{MPa}$ and $1,507.8 \mu$, respectively) tests, as shown in Table III.

Experimental results of the prosthesis. The weights of the condyle prostheses printed by FDM were $3.671 \mathrm{~g}$ (Model A) and $4.382 \mathrm{~g}$ (Model B). The compression test results indicated that the maximum forces that the prostheses were able to with-

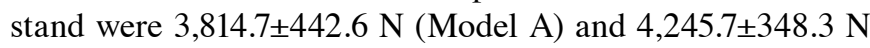
(Model B). The compressive force-displacement curves are presented in Fig. 6. 
A

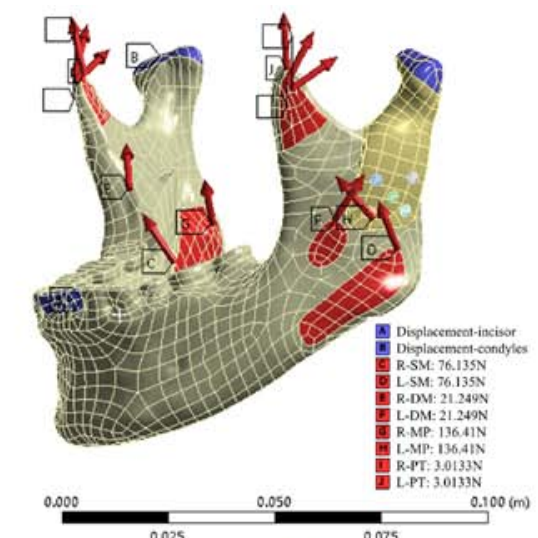

C

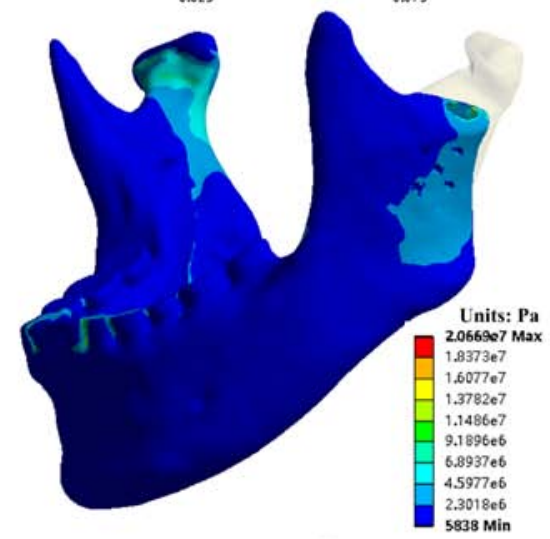

$\mathbf{E}$

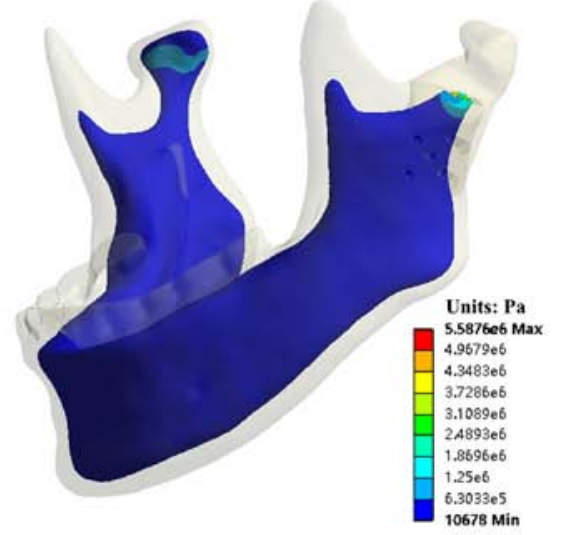

B

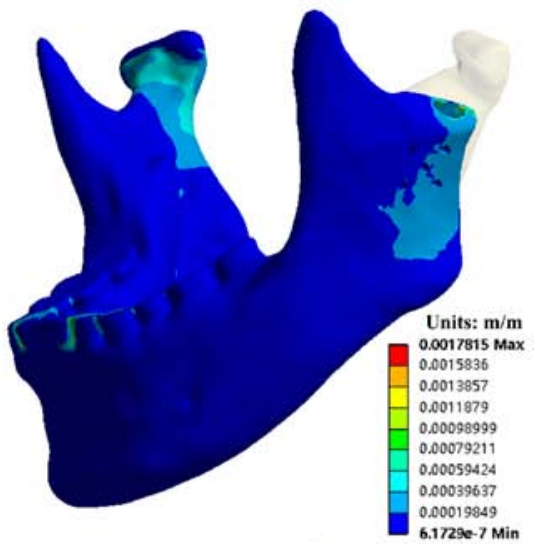

D

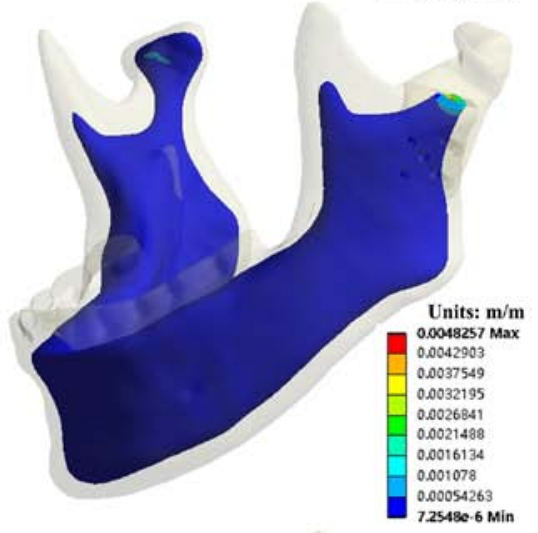

F

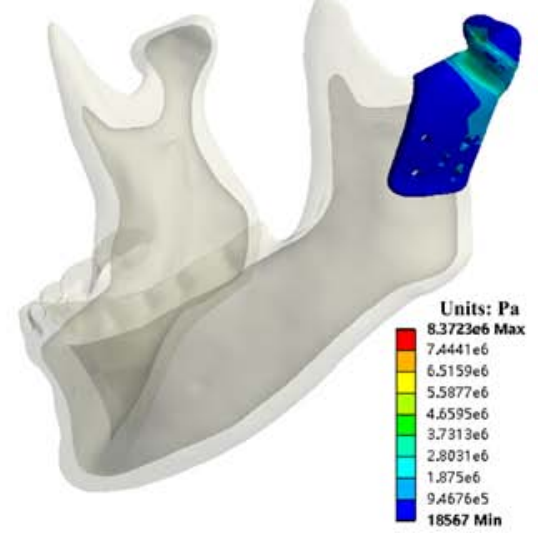

Figure 4. The von Mises stress and strain of bone and prosthesis in the INC task of model A. (A) INC Constraints; (B) von Mises strain and (C) von Mises stress of the entire cortical bone; (D) von Mises strain and (E) von Mises stress of the entire cancellous bone; (F) von Mises stress of the polyetheretherketone condylar prosthesis. INC, incisal clench.

\section{Discussion}

The present study introduced a novel TMJ prosthesis that differs from the existing prostheses from Biomet and TMJ Concepts. The custom condylar prostheses were designed using a reverse engineering approach and were prepared by $3 \mathrm{D}$ printing using PEEK as the printed material. PEEK is a high-strength semi-crystalline thermoplastic that was first introduced to the medical field in the early 1990s (18). PEEK has excellent biocompatibility and its yield strength and Young's modulus are closer to cortical bone than metal prostheses (13), which facilitates the design of implants that reduce stress shielding. Furthermore, high radiographic penetration may effectively avoid artifacts during post-operative examination (13). These advantages make PEEK a better candidate for loadbearing orthopedic devices, particularly in the spine.
However, reports on the use of PEEK in TMJ reconstruction are limited. In the present study, two PEEK condylar prosthesis models were designed to reflect the different degrees of surgical osteotomy. The FEM was used to analyze the biomechanical behavior of the condylar prostheses, which was validated through mechanical experiments.

To ensure the stability of the TMJ condylar prostheses, the stress of each component of the prosthesis should be less than the yield strength of the material. In the present study, consistent with the study of Kashi et al (19) from 2010, the stress in the condylar prostheses was predominantly concentrated in the anterior and posterior areas of the condylar neck. The maximum stress in this region was $10.733 \mathrm{MPa}$ and 8.5229 in Models A and B, respectively, which is far less than the yield strength of PEEK. Concerning the five bone screws used to fix the PEEK condylar prostheses, the maximum von Mises 

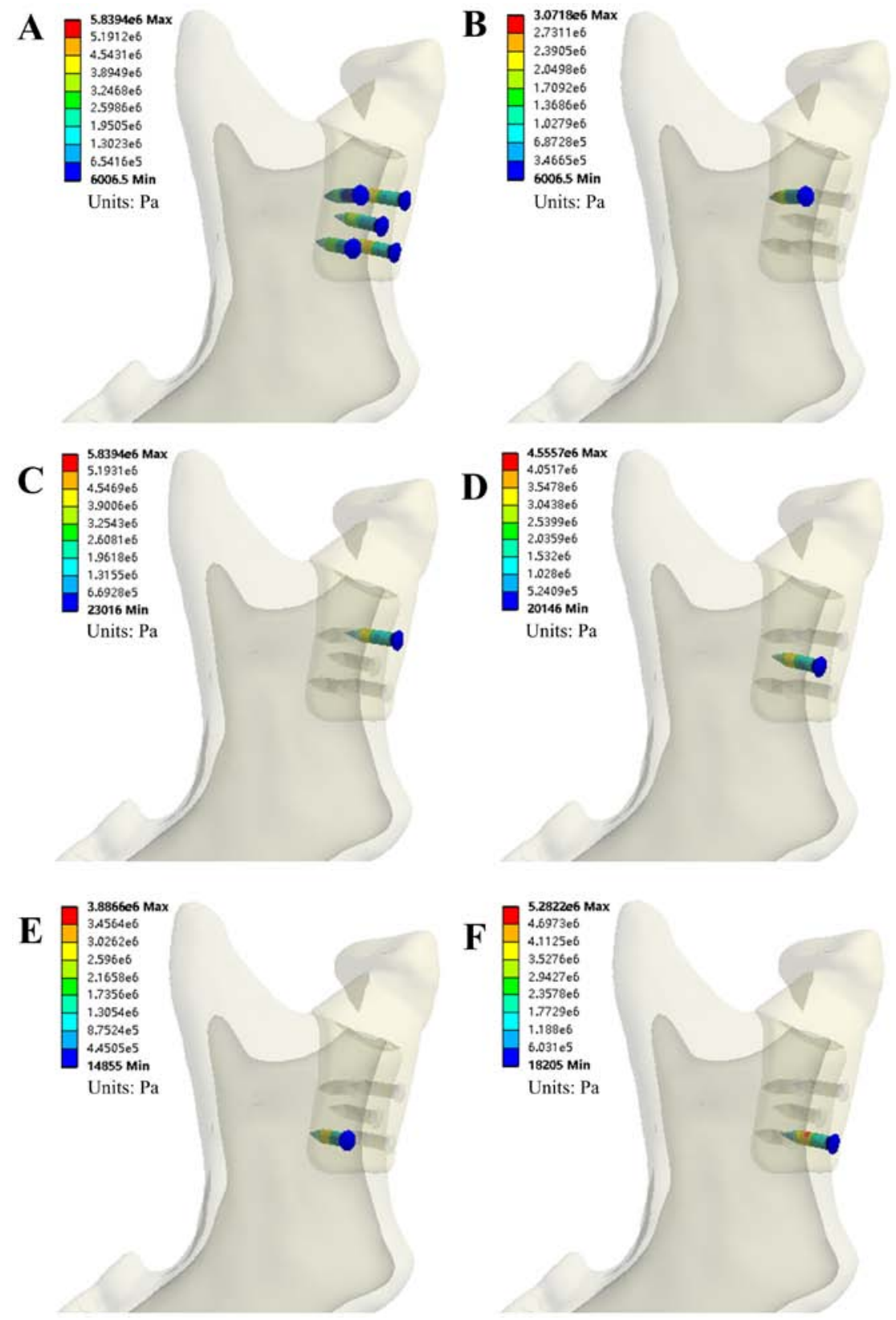

Figure 5. The von Mises stress of screws in the INC task of model A. (A) All five screws; (B) screw \#1; (C) screw \#2; (D) screw \#3; (E) screw \#4; and (F) screw \#5. INC, incisal clench.

stresses in models A and B were $9.7075 \mathrm{MPa}$ (screw \#5) and $2.7697 \mathrm{MPa}$ (screw \#2), far less than the yield strength of titanium alloy. The peak stresses of the personalized PEEK condyle prosthesis designed in this study and its retention screws are far less than the yield strength of their respective materials. Therefore, theoretically, the prostheses and screws would not fracture. Moreover, the stress values of these screws are significantly lower than the stress generated by a screw fixing metal condyle prosthesis $(17,20)$, it showed that the PEEK condyle prostheses reduced the risk of the retention screw fracture compared to the metal prostheses. In addition, consistent with the study of Kashi et al (19) from 2010, the peak von Mises stresses were observed in the upper and posterior regions of the mandible, demonstrating the importance of the number and location of screws when designing PEEK condyle prostheses.
The results of von Mises stress and strain generated by the four clenching tasks in the entire mandible have the following implications. First, the relationship between the von Mises strain and variations in bone mass were divided into the following ranges: disuse (bone loss): $<10 \mu \mathrm{m} / \mathrm{m}(21),<50 \mu \mathrm{m} / \mathrm{m}$ (22) or $<200 \mu \mathrm{m} / \mathrm{m}$ (23); normal load: $100-2,000 \mu \mathrm{m} / \mathrm{m}$; mild overload (bone gain): 2,000-4,000 $\mu \mathrm{m} / \mathrm{m}$ (24); pathologic overload (irreversible bone damage): $>4,000 \mu \mathrm{m} / \mathrm{m}$ (24); and bone fractures: $15,700-20,000 \mu \mathrm{m} / \mathrm{m}$ (24). With the exclusion of the right condyle neck (near the fixed and restrained position) and the lingual edge of the osteotomy surface (sharp edges after osteotomy), the maximum strain on cortical bone and cancellous bone was usually between 100 and $4,000 \mu \mathrm{m} / \mathrm{m}$. Furthermore, regarding the relationship between the von Mises stress and bone mass changes, a study by Sugiura et al (25) from 2000 indicated that the critical threshold for bone resorp- 
Table II. Simulation results of the four clenching tasks (Model A).

\begin{tabular}{|c|c|c|c|c|}
\hline \multirow[b]{2}{*}{ Component/evaluation parameter } & \multicolumn{4}{|c|}{ Maximum value } \\
\hline & INC & ICP & LMOL & RMOL \\
\hline \multicolumn{5}{|l|}{ Condylar prosthesis } \\
\hline von Mises stress (MPa) & 8.3723 & 10.733 & 4.8598 & 10.567 \\
\hline von Mises strain $(\mu)$ & $3,263.7$ & $4,261.5$ & $1,889.3$ & $4,027.2$ \\
\hline \multicolumn{5}{|l|}{ Cortical bone } \\
\hline von Mises stress (MPa) & 20.669 & 42.688 & 34.23 & 31.315 \\
\hline von Mises strain $(\mu)$ & $1,781.5$ & $4,897.2$ & $3,388.1$ & 2913.8 \\
\hline \multicolumn{5}{|l|}{ Cancellous bone } \\
\hline von Mises stress (MPa) & 5.5876 & 10.826 & 3.7015 & 6.792 \\
\hline von Mises strain $(\mu)$ & $4,825.7$ & $7,772.4$ & $2,920.5$ & $5,857.4$ \\
\hline \multicolumn{5}{|l|}{ All screws } \\
\hline von Mises stress (MPa) & 5.8394 & 9.7075 & 5.3554 & 9.4276 \\
\hline von Mises strain $(\mu)$ & 53.115 & 88.269 & 54.225 & 85.726 \\
\hline \multicolumn{5}{|l|}{ Screw \#1 } \\
\hline von Mises stress (MPa) & 3.0718 & 7.7425 & 4.7278 & 7.6845 \\
\hline von Mises strain $(\mu)$ & 27.937 & 87.112 & 54.225 & 78.165 \\
\hline \multicolumn{5}{|l|}{ Screw \#2 } \\
\hline von Mises stress (MPa) & 5.8394 & 9.5725 & 5.3554 & 9.4276 \\
\hline von Mises strain $(\mu)$ & 53.115 & 87.043 & 48.812 & 85.726 \\
\hline \multicolumn{5}{|l|}{ Screw \#3 } \\
\hline von Mises stress (MPa) & 4.5557 & 8.445 & 4.5214 & 7.6839 \\
\hline von Mises strain $(\mu)$ & 41.441 & 76.817 & 41.128 & 69.894 \\
\hline \multicolumn{5}{|l|}{ Screw \#4 } \\
\hline von Mises stress (MPa) & 3.8866 & 7.7507 & 4.2435 & 6.7267 \\
\hline von Mises strain $(\mu)$ & 35.34 & 70.501 & 38.600 & 61.188 \\
\hline \multicolumn{5}{|l|}{ Screw \#5 } \\
\hline von Mises stress (MPa) & 5.2822 & 9.7075 & 5.2122 & 9.2533 \\
\hline von Mises strain $(\mu)$ & 48.042 & 88.269 & 47.394 & 84.139 \\
\hline
\end{tabular}

The numbering of the screws is according to Fig. 5. INC, incisal clench; ICP, intercuspal position; L/RMOL, left/right unilateral molar clench.

tion should be approximately -50 MPa. In the simulations of the present study, the von Mises stress values in the bone were all $<50 \mathrm{MPa}$. In the present simulation of the four occlusal tasks, the stress and strain of the mandible after adding a PEEK condyle prosthesis were typically within the normal load range. Theoretically, no disuse and overload absorption of the mandible was expected. In addition, temporalis and part of the masseter muscles were resected in Model B; therefore, the maximum stress of the condyle and the stress and strain of the mandible were smaller than those in Model A.

Personalized 3D-printed PEEK condylar prostheses are able to meet individual requirements more effectively due to the simple preparation method, one-time molding, convenient processing and high material utilization. Furthermore, the intraoperative adaptability is expected to surpass that of already available TMJ prostheses. Furthermore, the experimental validation of the biomechanical properties of the developed prostheses suggested that the prostheses are able to withstand a maximum force of 3,814.7 $\pm 442.6 \mathrm{~N}$ (Model A) and 4,245.7 $\pm 348.3 \mathrm{~N}$ (Model B), which is much larger than the combined maximum muscle force in the $\mathrm{Z}$-axis direction in the ICP. Therefore, the 3D-printed PEEK condyle prosthesis developed in the present study is able to meet the clinical requirements of TMJ reconstruction in terms of its mechanical properties. This experiment revealed the following: FDM printing is accomplished by the accumulation of materials layer by layer and the molecular chains between the layers are less crossed and entangled, resulting in a small interlayer force. When designing the printing direction of the prosthesis, it is necessary to consider the entire force environment and characteristics of the prosthesis, so that frequent and larger forces act in the vertical plane direction rather than the interlayer direction.

As with any theoretical simulation study, there are certain limitations. First, a comparative study has yet to be performed between the custom PEEK prostheses and a standard prosthesis. In a future study, the stress and strain distribution between the PEEK condylar prosthesis and the Biomet 
Table III. Simulation results of the four clenching tasks (Model B).

Maximum value

Component/evaluation parameter

$\begin{array}{llll}\text { INC ICP } & \text { LMOL }\end{array}$

Condylar prosthesis

von Mises stress (MPa)

5.9419

6.7679

1.9178

8.5229

von Mises strain $(\mu)$

$2,382.8$

$2,710.4$

769.92

$3,551.8$

Cortical bone

von Mises stress (MPa)

13.93

34.535

43.038

24.148

von Mises strain $(\mu)$

$1,240.4$

$3,498.2$

$4,066.1$

$2,548.2$

Cancellous bone

von Mises stress (MPa)

2.5734

3.2374

2.4105

3.4237

von Mises strain $(\mu)$

$1,651.3$

$2,059.7$

$1,507.8$

$2,277.5$

All screws

von Mises stress (MPa)

1.8837

1.9063

0.65778

2.7697

von Mises strain $(\mu)$

17.267

17.441

6.041

25.332

Screw \#1

von Mises stress (MPa)

1.4413

1.3578

0.44294

1.6534

von Mises strain $(\mu)$

13.455

12.69

4.1499

15.338

Screw \#2

von Mises stress (MPa)

1.8837

1.9063

0.65778

2.7697

von Mises strain $(\mu)$

17.267

17.441

6.041

25.332

Screw \#3

von Mises stress (MPa)

1.3577

1.3148

0.44833

1.5328

von Mises strain $(\mu)$

12.48

12.089

4.0937

14.087

Screw \#4

von Mises stress (MPa)

0.9578

0.86554

0.2715

0.90107

von Mises strain $(\mu)$

8.8955

7.9824

2.5222

8.389

Screw \#5

von Mises stress (MPa)

1.3605

1.338

0.44003

1.7646

von Mises strain $(\mu)$

12.528

12.311

4.2029

16.225

The numbering of the screws is according to Fig. 5. INC, incisal clench; ICP, intercuspal position; L/RMOL, left/right unilateral molar clench.
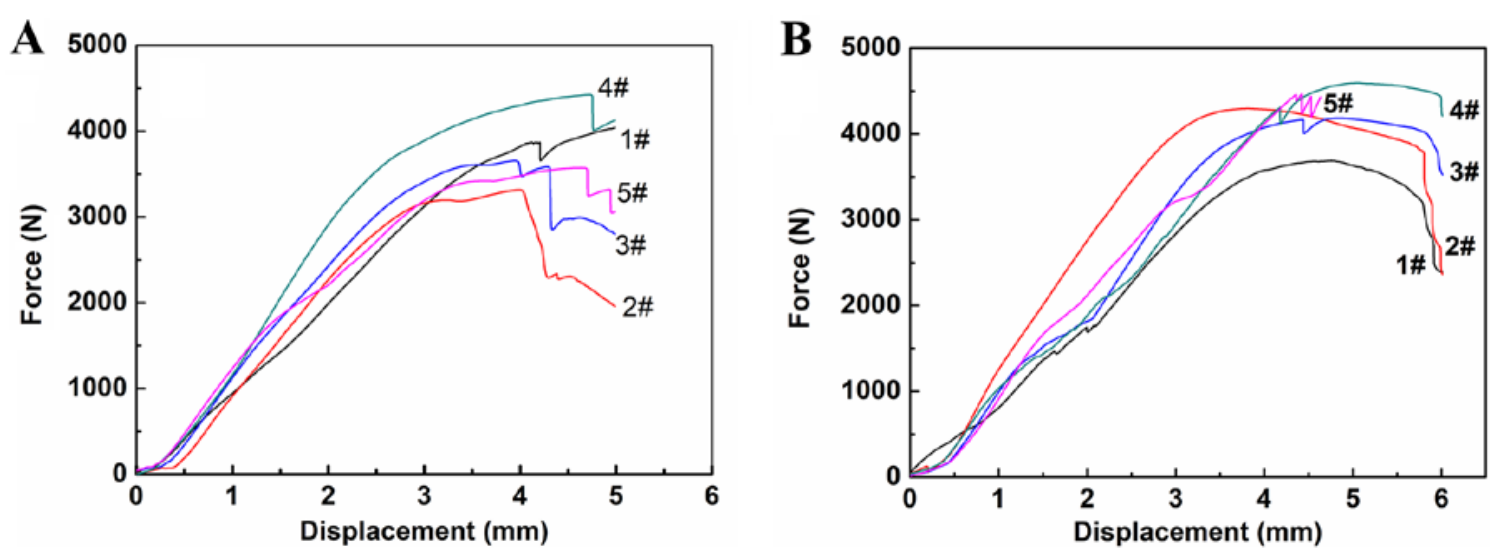

Figure 6. Compressive force-displacement curves for (A) Model A and (B) Model B. The numbering of the screws is according to Fig. 5.

stock prosthesis will be compared to further demonstrate the biomechanical advantages of the proposed device. In addition, a compression experiment will be conducted to evaluate the biomechanics of the prosthesis. In subsequent studies, fatigue 
and wear tests will be performed on the personalized PEEK condyle prosthesis using mechanical testing equipment and validation in animals.

\section{Acknowledgements}

Not applicable.

\section{Funding}

This work was supported by the National Natural Science Foundation of China (grant no. 81801034), the High-level Introduced Talent Fund Project of Xi'an Medical University (grant no. 2018RCYJ03), the Key R\&D Program of the Department of Science and Technology of Shaanxi Province (grant no. 2020SF-023) and the Key R\&D Program of Guangdong Province (grant no. 2018B090906001).

\section{Availability of data and materials}

The datasets used and/or analysed during the current study are available from the corresponding author on reasonable request.

\section{Authors' contributions}

$\mathrm{MH}$ and CL designed and supervised the project. FG, SH, CY and DL performed the experiments. FG, SH and CY analyzed the data. FG and $\mathrm{SH}$ confirmed the authenticity of the raw data. FG wrote the manuscript. All authors read and approved the final manuscript.

\section{Ethics approval and consent to participate}

The patient provided informed consent.

\section{Patient consent for publication}

The patient has signed an informed consent for the use of their CT data.

\section{Competing interests}

The authors declare that they have no competing interests.

\section{References}

1. Rodrigues YL, Mathew MT, Mercuri LG, da Silva JSP, Henriques B and Souza JCM: Biomechanical simulation of temporomandibular joint replacement (TMJR) devices: A scoping review of the finite element method. Int J Oral Maxillofac Surg 47: 1032-1042, 2018.

2. Jones R: The use of virtual planning and navigation in the treatment of temporomandibular joint ankylosis. Aust Dent J 58: 358-367, 2013.

3. Wolford LM, Dingwerth DJ, Talwar RM and Pitta MC: Comparison of 2 temporomandibular joint total joint prosthesis systems. J Oral Maxillofac Surg 61: 685-690, discussion 690, 2003.

4. Zhao Y, Wong SM, Wong HM, Wu S, Hu T, Yeung KW and Chu PK: Effects of carbon and nitrogen plasma immersion ion implantation on in vitro and in vivo biocompatibility of titanium alloy. ACS Appl Mater Interfaces 5: 1510-1516, 2013.

5. Niki Y, Matsumoto H, Otani T, Suda Y and Toyama Y: Metal ion concentrations in the joint fluid immediately after total knee arthroplasty. Mod Rheumatol 11: 192-196, 2001.
6. Wang L, He S, Wu X, Liang S, Mu Z, Wei J, Deng F, Deng Y and Wei S: Polyetheretherketone/nano-fluorohydroxyapatite composite with antimicrobial activity and osseointegration properties. Biomaterials 35: 6758-6775, 2014.

7. Sarot JR, ContarCM,Cruz AC and de Souza Magini R: Evaluation of the stress distribution in CFR-PEEK dental implants by the three-dimensional finite element method. J Mater Sci Mater Med 21: 2079-2085, 2010.

8. Jonkergouw J, van de Vijfeijken SE, Nout E, Theys T, Van de Casteele E, Folkersma H, Depauw PR and Becking AG: Outcome in patient-specific PEEK cranioplasty: A two-center cohort study of 40 implants. J Craniomaxillofac Surg 44: 1266-1272, 2016.

9. Lethaus B, Safi Y, ter Laak-Poort M, Kloss-Brandstätter A, Banki F, Robbenmenke C, Steinseifer U and Kessler P: Cranioplasty with customized titanium and PEEK implants in a mechanical stress model. J Neurotrauma 29: 1077-1083, 2012.

10. Zegers T, Ter Laak-Poort M, Koper D, Lethaus B and Kessler P: The therapeutic effect of patient-specific implants in cranioplasty. J Craniomaxillofac Surg 45: 82-86, 2017.

11. Hu B, Yang X, Hu Y, Lyu Q, Liu L, Zhu C, Zhou C and Song Y: The n-HA/PA66 cage versus the PEEK cage in anterior cervical fusion with single-level discectomy during 7 years of follow-up. World Neurosurg 123: e678-e684, 2019.

12. Zhang J, Pan A, Zhou L, Yu J and Zhang X: Comparison of unilateral pedicle screw fixation and interbody fusion with PEEK cage vs. standalone expandable fusion cage for the treatment of unilateral lumbar disc herniation. Arch Med Sci 14: 1432-1438, 2018.

13. Kurtz SM and Devine JN: PEEK biomaterials in trauma, orthopedic, and spinal implants. Biomaterials 28: 4845-4869, 2007.

14. Han ML, Li MH, Ji X, Han RY, Zhang N, Cui LH, Sun LF and Han CM: Establishment of TMJ defect models and evaluation on repair effect of CFR-PEEK material artificial joint in rabbits. J Jilin Univ Med Ed 43: 903-909, 2017.

15. Arabshahi Z, Kashani J, Rafiq M, Kadir A and Azari A: Influence of the TMJ implant geometry on stress distribution. Adv Mat Res 488: 991-995, 2012.

16. Hsu JT, Huang HL, Tu MG and Fuh LJ: Effect of bone quality on the artificial temporomandibular joint condylar prosthesis. Oral Surg Oral Med Oral Pathol Oral Radiol Endod 109: e1-e5, 2010.

17. Huang HL, Su KC, Fuh LJ, Chen MY, Wu J, Tsai MT and Hsu JT: Biomechanical analysis of a temporomandibular joint condylar prosthesis during various clenching tasks. J Craniomaxillofac Surg 43: 1194-1201, 2015.

18. Torstrick FB, Lin ASP, Potter D, Safranski DL, Sulchek TA, Gall K and Guldberg RE: Porous PEEK improves the bone-implant interface compared to plasma-sprayed titanium coating on PEEK. Biomaterials 185: 106-116, 2018.

19. Kashi A, Chowdhury AR and Saha S: Finite element analysis of a TMJ implant. J Dent Res 89: 241-245, 2010.

20. Chen X, Wang Y, Mao Y, Zhou Z, Zheng J, Zhen J, Qiu Y, Zhang S, Qin $\mathrm{H}$ and Yang C: Biomechanical evaluation of Chinese customized three-dimensionally printed total temporomandibular joint prostheses: A finite element analysis. J Craniomaxillofac Surg 46: 1561-1568, 2018.

21. Rubin CT and Lanyon LE: Regulation of bone mass by mechanical strain magnitude. Calcif Tissue Int 37: 411-417, 1985.

22. Jaworski ZF, Liskova-Kiar M and Uhthoff HK: Effect of long-term immobilisation on the pattern of bone loss in older dogs. J Bone Joint Surg Br 62-B: 104-110, 1980.

23. Lovell NC: Structure function, and adaptation of compact bone. By R. Bruce Martin and David B. Burr. Am J Phys Anthropol 82: 116-117, 1990.

24. Mellal A, Wiskott HWA, Botsis J, Scherrer SS and Belser UC: Stimulating effect of implant loading on surrounding bone. Comparison of three numerical models and validation by in vivo data. Clin Oral Implants Res 15: 239-248, 2004.

25. Sugiura T, Horiuchi K, Sugimura M and Tsutsumi S: Evaluation of threshold stress for bone resorption around screws based on in vivo strain measurement of miniplate. J Musculoskelet Neuronal Interact 1: 165-170, 2000.

This work is licensed under a Creative Commons Attribution-NonCommercial-NoDerivatives 4.0 International (CC BY-NC-ND 4.0) License. 